TITLE:

\title{
Photothermal fabrication of microstructures in transparent low- melting media doped with rare earth ions as a light absorber
}

\section{$\operatorname{AUTHOR}(\mathrm{S})$ :}

Takahashi, M; Saito, M; Mizuno, M; Kakiuchida, H; Tokuda, Y; Yoko, T

\section{CITATION:}

Takahashi, M ...[et al]. Photothermal fabrication of microstructures in transparent lowmelting media doped with rare earth ions as a light absorber. APPLIED PHYSICS LETTERS 2006, 88(19): 191914.

\section{ISSUE DATE:}

2006-05-08

URL:

http://hdl.handle.net/2433/50339

\section{RIGHT:}

Copyright 2006 American Institute of Physics. This article may be downloaded for personal use only. Any other use requires prior permission of the author and the American Institute of Physics. 


\title{
Photothermal fabrication of microstructures in transparent low-melting media doped with rare earth ions as a light absorber
}

\author{
Masahide Takahashia) \\ Institute for Chemical Research, Kyoto University, Uji, Kyoto, 611-0011, Japan and PRESTO, Japan \\ Science and Technology Agency (JST), 4-1-8 Honcho, Kawaguchi, Saitama 332-0012, Japan \\ Masanori Saito, Megumi Mizuno, Hiroshi Kakiuchida, Yomei Tokuda, and Toshinobu Yoko \\ Institute for Chemical Research, Kyoto University, Uji, Kyoto, 611-0011, Japan \\ (Received 15 December 2005; accepted 14 April 2006; published online 10 May 2006)

\begin{abstract}
Microstructures were fabricated by a photothermal process in a low-melting organic-inorganic hybrid material of tin silicophosphate doped with $\mathrm{Nd}^{3+}$ ion as a light absorber. The negative refractive index change of the order of $10^{-3}$ was photothermally induced inside the low-melting glass with a spatial resolution ranging from 10 to $100 \mu \mathrm{m}$ using a $514.5 \mathrm{~nm}$ emission from an $\mathrm{Ar}^{+}$ laser of less than $30 \mathrm{~mW}$ as the excitation source. The volume expansion due to the photogenerated heat at the laser focus is found to be responsible for the photothermally induced negative photorefractive index change. (C) 2006 American Institute of Physics. [DOI: 10.1063/1.2203516]
\end{abstract}

Transparent materials with a periodic modulation of the refractive index can be used for two-dimensional (2D) or three-dimensional (3D) photonic devices that control the light wave for optical information processing. ${ }^{1}$ Many techniques to fabricate microstructures including photolithography, focused ion beam, lithography, electroplating, and molding, etc., have been practically utilized to make such devices. ${ }^{2}$ The femtosecond laser fabrication of transparent materials through multiphoton absorption is also attractive for producing microstructures. ${ }^{3-5}$ The laser direct writing technique using ultraviolet laser sources is a facile way to make relatively simple telecommunication devices. ${ }^{6,7} \mathrm{Re}-$ cently, the photoinduced thermal fabrication of a micro/ nanostructure has been reported by several groups. ${ }^{8-10}$ The photoinduced crystallization of nonconventional metal oxide glasses was reported to fabricate microstructures made of nonlinear optical crystals. ${ }^{8}$ The formation of a nanostructure involving the laser induced expansion of the material has also been reported. ${ }^{9}$ The laser welding technique of thermoplastic polymers was achieved by a laser melting technique. ${ }^{10}$ The use of the photothermal process is believed to offer an easy way to fabricate microstructures in lowmelting media.

In the present study, the photothermally induced photorefractive index change in doped low-melting transparent media, such as organic-inorganic hybrid silicophosphate glasses, ${ }^{11-13}$ is demonstrated. Heat generation by the nonradiative phonon emission of excited rare earth ions doped in the glasses drives the photorefractive index change. A lowpower density laser beam can be used as a heat generation source resulting in the photothermally induced refractive index change of the low-melting materials. In addition, microstructures written in the matrix can be erased by a postheat-treatment or laser irradiation. Such a lower energy fabrication seems quite environmental friendly.

Low-melting organic-inorganic hybrid glassy materials of tin(II) methyl-modified silicophosphate glasses are used for the transparent low-melting media. ${ }^{11}$ The nominal composition of the starting materials was

\footnotetext{
${ }^{a)}$ Electronic mail: masahide@noncry.kuicr.kyoto-u.ac.jp
}

$\mathrm{Me}_{2} \mathrm{SiCl}_{2}: \mathrm{SnCl}_{2}: \mathrm{H}_{3} \mathrm{PO}_{3}: \mathrm{NdCl}_{3} \cdot 6 \mathrm{H}_{2} \mathrm{O}=0.75: 1: 1: 0.02$. The glass was doped with rare earth ion, $\mathrm{Nd}^{3+}$ ion $(0.7 \%$ in cationic ratio), as a light absorber. Laser fabrication was carried out using an $\mathrm{Ar}^{+}$laser in the cw operation mode of which the light intensity was less than $30 \mathrm{~mW}$. The writing dynamics was investigated by in situ Mach-Zehnder interferometry (MZI).

Preparation of the tin(II) methyl-modified silicophosphite glasses was reported elsewhere. ${ }^{11,12}$ The resultant molten glass was poured into a Pyrex cuvette in order to obtain an optically smooth surface for laser irradiation. The glass transition temperature of the hybrid glass was estimated to be $26^{\circ} \mathrm{C}$. The coefficients of thermal expansion were estimated to be $4 \times 10^{-4}$ and $2 \times 10^{-5} \mathrm{~K}^{-1}$ above and below the glass transition temperature, respectively. $\mathrm{An} \mathrm{Ar}^{+}$laser of $514.5 \mathrm{~nm}$ radiation (Spectra Physics Co., USA) was used as the excitation source. Figure 1(a) shows the present optical setup for the laser fabrication. A collimated beam was focused on the interior of the material to be fabricated using a $20 \times$ objective lens $[f=9 \mathrm{~mm}$, numerical aperture (NA) $=0.3$ or $f=2 \mathrm{~mm},(\mathrm{NA})=0.4]$. The modified region was directly observed by an optical microscope after the irradiation. The photoinduced refractive index change was estimated by in situ and ex situ MZI. Figure 1(b) shows the

(a)

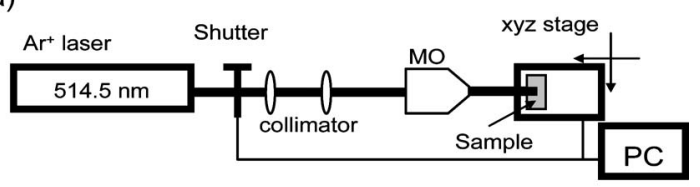

(b)

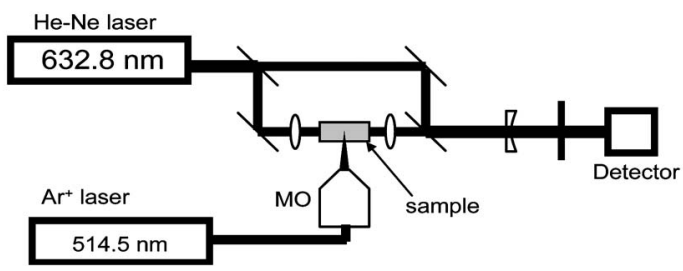

FIG. 1. Optical setups: (a) the photothermal laser writing and (b) in-situ MZI. 


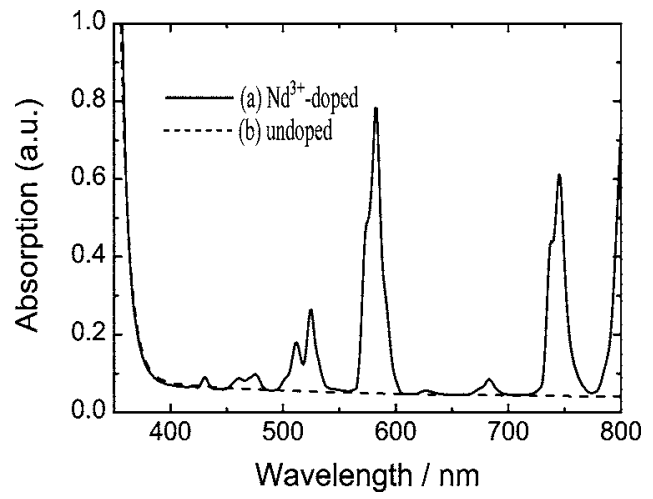

FIG. 2. Optical absorption spectra of (a) $\mathrm{Nd}^{3+}$-doped and (b) undoped tin(II) silicophosphate glasses.

optical setup of the in situ MZI. He-Ne laser $(632.8 \mathrm{~nm})$ was used as the probe light source. The objective light was focused on the irradiated region by a convex lens $(f=50)$ to observe the refractive index change. In a case of the ex situ MZI, the writing beam was turned off. A circular fringe pattern was observed in order to determine the sign of the refractive index change in terms of the direction of the fringe ring movement.

Figure 2 shows the optical absorption spectra of the doped and undoped glasses. Several sharp absorption bands corresponding to the $\mathrm{Nd}^{3+} f-f$ transitions are seen in the spectrum of the doped glass. When the doped glass was excited at $514.5 \mathrm{~nm}$, the photoluminescence band is observed at $536 \mathrm{~nm}$. The excitation energy is emitted to the surroundings through nonradiative decay process from the ${ }^{4} G_{11 / 2}$ to ${ }^{2} G_{7 / 2}$ states as phonons, namely, heat. ${ }^{14}$ Because the hybrid glasses exhibit low-melting temperatures less than $100^{\circ} \mathrm{C}$, it is possible to melt them locally by the laser irradiation (heating). Figure 3 shows the irradiation time dependence of the phase shift estimated by the in situ MZI, which corresponds to the change in the optical path length. The irradiated laser power was less than $10 \mathrm{~mW}(20 \times$ objective lens, $\mathrm{NA}=0.3)$, which was below the energy threshold of the persistent laser fabrication. As can be clearly seen from Fig. 3, the phase shift logarithmically developed. Although the several processes participated in the photothermal process, the change in the optical path length varied almost linearly with the irradiation power (inset of Fig. 3).

Assuming that the region subjected to the refractive index change is uniform and cylindrical in shape with a diam-

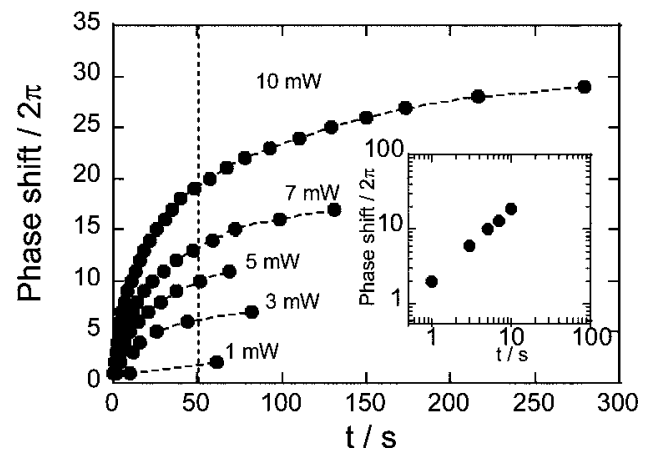

FIG. 3. In situ MZI during laser irradiation (MO: $f=2 \mathrm{~mm}, \mathrm{NA}=0.4$, irradiation time: $20 \mathrm{~min}$ ). The laser power was less than the fabrication threshold of the persistent structures. The inset shows the log-log relationship between the laser power and the phase shift.

Downloaded 05 Mar 2008 to 130.54.110.22. Redistribution subject
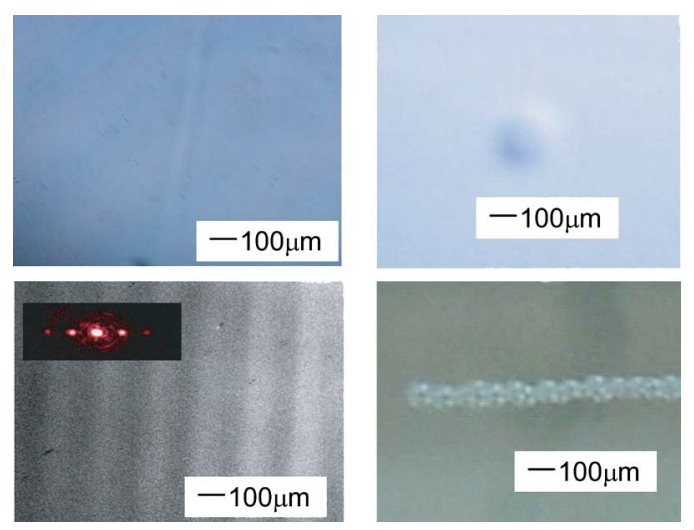

FIG. 4. (Color online) Optical microscope images of fabricated structures: (a) line (20 mW, MO: $f=2 \mathrm{~mm}, \mathrm{NA}=0.4$, irradiation time: $20 \mathrm{~min}$ ), (b) dot (20 mW, MO: $f=2 \mathrm{~mm}, \mathrm{NA}=0.4$, irradiation time: $15 \mathrm{~min}$ ), (c) phase gratings (ten lines were fabricated at $150 \mu \mathrm{m}$ intervals, each line was written by $20 \mathrm{~mW}, \mathrm{MO}: f=2 \mathrm{~mm}, \mathrm{NA}=0.4$, irradiation time: $15 \mathrm{~min}$ ) and inset shows the diffraction image of the $\mathrm{He}-\mathrm{Ne}$ laser light by the grating, and (d) array of voids ( $40 \mathrm{~mW}$, MO: $f=2 \mathrm{~mm}, \mathrm{NA}=0.4$, laser beam was scanning at $6.7 \mu \mathrm{m} / \mathrm{s})$.

eter of around $70 \mu \mathrm{m}$ (picture is shown later), the phase shift can be converted into the refractive index change. The estimated refractive index change under laser irradiation $(10 \mathrm{~mW})$ at the saturated phase shift is $\sim 0.05$. It is well known that the thermo-optic coefficient is expressed as ${ }^{15}$

$$
\frac{d n}{d T}=\frac{\left(n^{2}-1\right)\left(n^{2}+1\right)}{6 n}(\phi-\beta),
$$

where $\phi$ is the temperature coefficient of the electron polarizability and $\beta$ is the thermal expansion coefficient. For the organic-inorganic hybrid siloxane materials, $d n / d T$ largely depends on $\beta$ rather than $\phi$ because the former is much larger than the latter. ${ }^{16}$ The refractive index change $\Delta n$ and $\beta$ can be correlated by the Lorentz-Lorenz (LL) relationship

$$
\Delta n=-\frac{\left(n^{2}+2\right)\left(n^{2}-1\right)}{6 n}\left[1-\frac{V \Delta R}{R \Delta V}\right] \frac{\Delta V}{V},
$$

where $R$ is the molar refraction, $V$ the molar volume, and $(\Delta V / \Delta T) / V=3(\Delta L / \Delta T) / L=3 \beta$. Therefore, volume change of $\sim 3.8 \%$ results in the refractive index change of 0.05 . We can then estimate the temperature change at the laser focus. For example, the temperature rises to be around $100{ }^{\circ} \mathrm{C}$ under steady state irradiation of $10 \mathrm{~mW}$ laser through a microobjective of $f=9 \mathrm{~mm}, \mathrm{NA}=0.3$.

As shown in Fig. 4, line, dot, phase gratings, and an array of voids in the micrometer range could be fabricated inside the glasses. The former two kinds of structures were written at a laser power of $20 \mathrm{~mW}$. It took 20 and $15 \mathrm{~min}$ to fabricate line and dot, respectively. Although the diameter of the laser beam waist is estimated to be around $5 \mu \mathrm{m}$, that of the written line was around $70 \mu \mathrm{m}$ as shown in Fig. 4. This broadening is explained by the thermal diffusion from the laser focus to the surroundings. The thinner line, with a diameter of around $10 \mu \mathrm{m}$, could be fabricated using a higher NA micro-objective $(f=2 \mathrm{~mm}, \mathrm{NA}=0.4$, laser power: $10 \mathrm{~mW}$ ). The phase gratings can also be fabricated inside the glass as shown in Fig. 4(c). The amplitude of the refractive index modulation was estimated as $3 \times 10^{-3}$ by assuming the Raman-Nas-type grating. ${ }^{17}$ On increasing the laser power $(>40 \mathrm{~mW})$, an array of voids could be made inside the
AIP license or copyright; see http://apl.aip.org/apl/copyright.jsp 
(a)
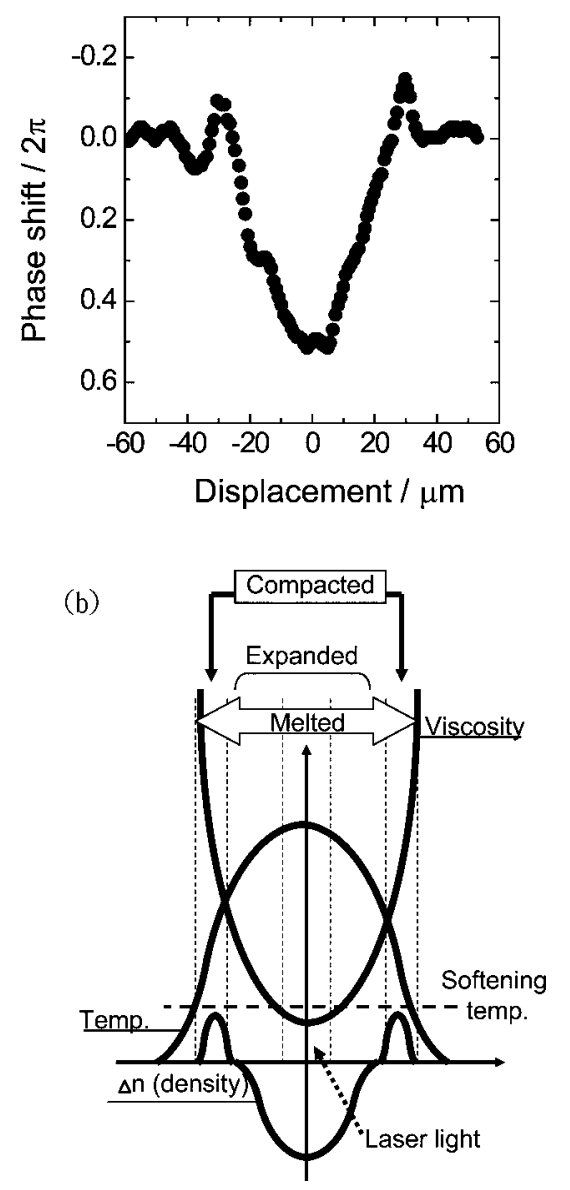

FIG. 5. (a) Cross-sectional distribution of phase shift for the line structure shown in Fig. 3(a) and (b) schematic illustration of photothermal process taking place in the doped media.

glasses. By applying an electric field during the laser irradiation, it was possible to induce the optically anisotropic structures in the present glass. The induced refractive index changes could be erased by the post-heat-treatment above $\sim 50{ }^{\circ} \mathrm{C}$ and rewritten by the subsequent laser irradiation.

The fabricated structures [Fig. 4(a)] remained unchanged for more than several months. The refractive index change by the laser irradiation was estimated by ex situ MZI. Figure 5 shows the cross-sectional distribution of the phase shift of the line shown in Fig. 4, which was estimated by scanning the line structure across the focused probe beam of the MZI. The sign of the refractive index change was estimated by the direction of the fringe ring movement. The negative index change of $-2.0 \times 10^{-3}$ was induced at the center of the line structure. Based on the LL relationship, the volume expansion at the focus was estimated to be $\sim 0.15 \%$ indicating that the corresponding temperature of the frozen-in structure of fabricated region, the so-called fictive temperature, is $\sim 40 \mathrm{~K}$ higher than that of the surroundings. At the periphery of the line, a positive index change was observed. These results indicate that the refractive index change was caused by the expansion of the glass at the laser focus, and hence the densification occurred at the periphery of the heated region, which is depicted in Fig. 5(b). Consequently, we can say that a persistent photorefractive index change could be induced by irradiating a low-power density cw laser light at a wavelength that should be well matched with the optical absorption band of the doped light absorbers.

In conclusion, we can fabricate microstructures inside low-melting transparent media doped with light absorbers by a photothermal process. Several microstructures of which the size was in the micrometer range from 10 to $100 \mu \mathrm{m}$ were fabricated using a cw $\mathrm{Ar}^{+}$laser $(<30 \mathrm{~mW})$ as the excitation source. The negative refractive index change could be induced by the thermal expansion of the irradiated part, and consequently, a positive index change was observed at its periphery. The obtained structure remained unchanged for several months under ambient conditions. The present photothermal fabrication technique for low-melting media could be applicable for low-melting glasses, thermoplastic polymers, etc. It was also confirmed by the present authors that an organic dye would also be useful as light absorbers. Such a lower energy fabrication seems quite environmentally friendly.

This work is partly supported by the Grants-in-Aid from the Ministry of Education, Science, Sports, and Culture, Japan, Nos. 16686041 and 13305061. One of the authors (M.T.) acknowledges the financial support from The Murata Science Foundation.

${ }^{1}$ S. G. Johnson and J. D. Joannopouos, Photonic Crystal The Road Map from Theory to Practice (Kluwer Academic, Boston, 2002), Chap. 1.

${ }^{2}$ E.-B. Kley, L.-C. Wittig, and A. Tünnermann, Microoptics (Springer, New York, 2004), Chap. 1.

${ }^{3}$ K. M. Davis, K. Miura, N. Sugimoto, and K. Hirao, Opt. Lett. 21, 1729 (1996).

${ }^{4}$ E. N. Glezer, M. Milosavljevic, L. Huahng, R. J. Frey, T.-H. Her, J. P. Callan, and E. Mazur, Opt. Lett. 21, 20023 (1996).

${ }^{5}$ H. B. Sun, S. Matsuo, and H. Misawa, Appl. Phys. Lett. 74, 786 (1999).

${ }^{6}$ M. Takahashi, A. Sakoh, K. Ichii, T. Tokuda, T. Yoko, and J. Nishii, Appl. Opt. 42, 4594 (2003).

${ }^{7}$ J. Nishii, K. Kintaka, H. Nishiyama, and M. Takahashi, J. Non-Cryst. Solids 326\&327, 464 (2003).

${ }^{8}$ R. Sato, Y. Benino, T. Fujiwara, and T. Komatsu, J. Non-Cryst. Solids 289, 228 (2001).

${ }^{9}$ M. Kuwahara, J. H. Kim and J. Tominaga, Microelectron. Eng. 73-74, 69 (2004).

${ }^{10}$ V. A. Kagan and G. P. Pinho, J. Reinf. Plast. Compos. 23, 95 (2004).

${ }^{11}$ H. Niida, M. Takahashi, T. Uchino, and T. Yoko, Phys. Chem. Glasses 43C, 416 (2002).

${ }^{12}$ H. Niida, M. Takahashi, T. Uchino, and T. Yoko, J. Mater. Res. 18, 1081 (2003).

${ }^{13}$ M. Takahashi, H. Niida, Y. Tokuda, and T. Yoko, J. Non-Cryst. Solids 326, 524 (2003).

${ }^{14} \mathrm{R}$. Reisfeld and C. K. Jørgensen, in Handbook of the Physics and Chemistry of Rare Earths, edited by K. A. Gschneider, Jr. and L. Eyring (Elsevier, Amsterdam, 1987), Vol. 9.

${ }^{15}$ L. Prod'homme, Phys. Chem. Glasses 4, 119 (1960).

${ }^{16}$ E. S. Kang, T. H. Lee, and B. S. Bae, Appl. Phys. Lett. 81, 1438 (2002).

${ }^{17}$ W. R. Klein and Bill D. Cook, IEEE Trans. Sonics Ultrason. 14, 123 (1967). 